\title{
Las rúbricas como herramienta de mejora del aprendizaje de la Termodinámica Técnica ${ }^{1}$
}

\section{Begoña Peña ${ }^{a}$}

a Universidad de Zaragoza - Departamento de Ingeniería Mecánica (España) e-mail: bpp@unizar.es.

\begin{abstract}
Promoted by the European Higher Education Area, new methodologies and pedagogical models have been assessed to improve learning outcomes and competences acquisition. Such models involve a wide variety of activities for learning and assessment purposes, but in many cases the student is not aware of the relationship between such activities and the final evaluation. This fact decreases motivation, worsening the learning process.

Rubrics are a powerful tool to these respects, as they provide to the student clear assessment criteria, tools for self-evaluation, immediate feedback about lacks and suggestions to improve. For the instructor, the design of rubrics obliges to be reflective about the coherence of learning activities with the competences, which leads to an improvement of the learning process

In the present work, quantitative and automated rubrics have been designed for the subject of Engineering Thermodynamics. Each criteria and level is related to certain questions and calculations accomplished by the student in Moodle platform. The methodology followed in the present academic course is here presented and analyzed to be enhanced in next courses.
\end{abstract}

Keywords: Rubrics, Formative Evaluation, Self-assessment, Higher Education Innovation, Engineering Thermodynamics.

\section{Resumen}

El Espacio Europeo de Educación Superior ha promovido la implantación de nuevas metodologías y modelos pedagógicos para mejorar los resultados de aprendizaje y la adquisición de competencias. Dichos modelos conllevan una amplia variedad de actividades de aprendizaje y evaluación, pero en muchos casos el estudiante no es consciente de la relación que existe entre dichas actividades y la evaluación final. Esto disminuye la motivación, empeorando el proceso de aprendizaje.

Las rúbricas son una herramienta potente a este respecto, porque proporcionan al estudiante criterios de evaluación claros, herramientas para autoevaluarse, realimentación inmediata sobre sus carencias y sugerencias para mejorar. Por otra parte, el diseño de rúbricas obliga al profesor a

\footnotetext{
${ }^{1}$ Este trabajo se ha desarrollado dentro del proyecto PIIDUZ_18_102 de innovación docente (Programa de Incentivación de la Innovación Docente del Vicerrectorado de Política Académica de la Universidad de Zaragoza).
} 
reflexionar sobre la coherencia entre las actividades de aprendizaje y las competencias, lo que conduce a una mejora del proceso de aprendizaje.

En el presente trabajo, se han diseñado rúbricas cuantitativas y automatizadas para la asignatura de Termodinámica Técnica. Cada criterio y nivel se asocia con ciertas preguntas y cálculos realizados por el estudiante en la plataforma Moodle. En este trabajo se presenta y analiza la metodología seguida en el presente curso académico y se extraen conclusiones para mejorarla.

Palabras clave: Rúbricas, Evaluación formativa, Autoevaluación, Innovación en Docencia Universitaria, Termodinámica Técnica.

\section{Introducción}

El diseño de evaluaciones formativas que requieran una inversión de tiempo aceptable es un desafío para los profesores, ya que idealmente la evaluación debe ser continua, personalizada en lo posible y adaptada a cada asignatura particular. Desde este punto de vista, en el presente trabajo se ha planteado el diseño de procedimientos de evaluación alternativos en base a los siguientes aspectos: (i) la evaluación debe ser una herramienta de aprendizaje; (ii) enfocada en la adquisición de competencias; (iii) con criterios claros conocidos de antemano por el alumno; (iv) con realimentación lo más inmediata posible sobre las carencias y errores y con sugerencias para mejorar; (v) incluyendo herramientas que permitan a los estudiantes evaluar por sí mismos su progreso en el aprendizaje; (vi) y debe proporcionar al profesor información continua sobre los avances de los estudiantes para corregir a tiempo las desviaciones que puedan aparecer.

Las rúbricas son herramientas de evaluación definidas como "documentos que articulan las expectativas para una tarea enumerando los criterios de evaluación y describiendo los niveles de calidad en relación a cada criterio" (Panadero, 2013). Para definir correctamente una rúbrica, se necesitan tres características esenciales: criterios de evaluación, niveles y descriptores (Dornish, 2006). Los criterios son las competencias a ser evaluadas; los niveles de desempeño son etiquetas para distinguir la calidad de los logros; los descriptores son un texto específico para cada nivel y criterio que describe lo que el estudiante debe saber hacer en cada caso.

Existen muchos ejemplos en la bibliografía sobre su aplicación en todos los niveles educativos (Reddy, 2010; Panadero, 2013) con diferentes propósitos: evaluación, aprendizaje, información sobre el progreso para el alumno y para el profesor, o autoevaluación, entre otros. Acercar la evaluación al estudiante es importante para ayudarlo a comprender los objetivos del aprendizaje y los criterios y niveles exigidos para conseguirlos. Disponer de esta información de antemano le permite reflexionar sobre su propio aprendizaje, sobre el nivel alcanzado y sobre cómo podría mejorar. Las rúbricas encajan perfectamente en este papel si se diseñan y se aplican correctamente. 
Las rúbricas pueden tener un carácter holístico o analítico (Dornish, 2006). Las primeras ofrecen una imagen global del aprendizaje que es útil y rápida desde el punto de vista del profesor, ya que son rápidas de implementar y de utilizar. Sin embargo, las rúbricas holísticas proporcionan poca información a los estudiantes y tienen muy poca utilidad como herramienta de aprendizaje. Las rúbricas analíticas separan la evaluación en criterios específicos, proporcionando información detallada sobre el proceso de aprendizaje, con recomendaciones útiles para mejorar. Obviamente, estas rúbricas requieren más tiempo que las holísticas, tanto en la etapa de diseño como durante su implantación, pero son mucho más útiles para el estudiante.

Existen muchas herramientas disponibles en Internet para desarrollar rúbricas: bancos de rúbricas prediseñadas, plantillas de rúbricas y generadores de rúbricas que permiten mayor flexibilidad para editar y crear rúbricas personalizadas (Dornish, 2006).

También los Entornos Virtuales de Aprendizaje (VLE), como Moodle, incluyen un editor de rúbricas que facilita la definición de los criterios, la descripción y el diseño de la rúbrica en sí misma (Moodle 2019). Para evaluar el trabajo de cada estudiante con estas rúbricas tradicionales, el profesor debe completar a mano una tabla con las calificaciones para cada criterio (Figura 1), lo que requiere dedicar bastante tiempo a esta tarea.

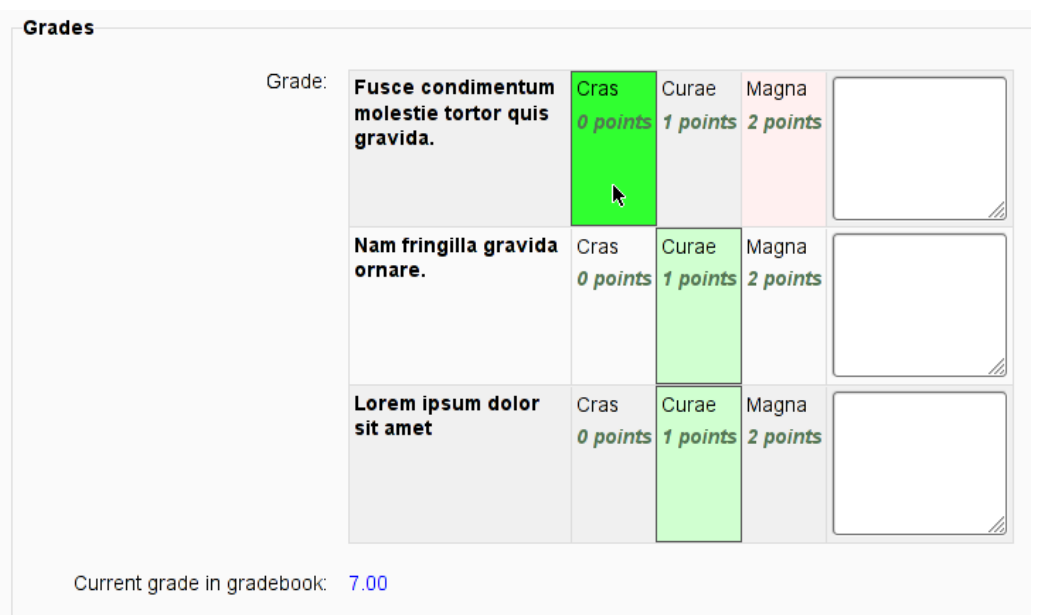

Fig. 1 Ejemplo de rúbrica en la plataforma Moodle (Moodle, 2019).

Por tanto, las rúbricas son herramientas muy apropiadas para conseguir una evaluación formativa pero, desde el punto de vista del profesor, su uso conlleva una dedicación importante, tanto en la fase de diseño como en la fase de utilización. Para grupos números de estudiantes, esto supone una inversión de tiempo inasumible, dadas atribuciones profesionales del profesorado universitario (dirección, desarrollo y gestión de la investigación, atención de alumnos, gestión universitaria, transferencia de conocimiento,...). 
Para ciertos campos de conocimiento, como los relacionados con las ciencias sociales y humanas, las actividades de evaluación se basan generalmente en desarrollar trabajos artísticos o ensayos. En tales casos, es muy difícil desarrollar rúbricas automatizadas y la única opción para usar rúbricas formativas es completarlas a mano para cada alumno. Sin embargo, para áreas tecnológicas, científicas y de salud, las preguntas cuantitativas o de respuesta corta se usan habitualmente en la evaluación y se pueden implementar en VLE para obtener la calificación de forma automática. Estos registros se pueden utilizar para desarrollar rúbricas cuya evaluación sea cuantitativa y automatizada.

Este trabajo se ha realizado dentro del proyecto de innovación docente "Combinando el modelo de aula inversa con el uso de TIC y metodologías activas", cuyo objetivo final es mejorar los resultados de aprendizaje y las competencias adquiridas. Desde el punto de vista del profesor, la definición de rúbricas ha servido para reflexionar sobre la coherencia entre las actividades de aprendizaje recomendadas para seguir la evaluación continua y las competencias que el estudiante debe adquirir. Desde el punto de vista del estudiante, las rúbricas le dan información clara y detallada de lo que se espera de él y le ayudan a autoevaluarse y autorregularse.

Concretamente, se presenta el procedimiento para definir rúbricas automatizadas y cuantitativas, basadas en las actividades de evaluación de Moodle, y se realiza un análisis objetivo sobre su utilidad, tanto desde el punto de vista del profesor, como del alumno.

\section{Objetivos}

El trabajo aquí presentado ha tenido como objetivo general evaluar tanto la factibilidad de desarrollar rúbricas cuantitativas y automatizadas, como su utilidad para la evaluación formativa del estudiante y para evaluar la coherencia entre las competencias y las actividades de aprendizaje.

Los objetivos específicos han sido:

- Definir las rúbricas: criterios, niveles y descriptores.

- Relacionar las preguntas de las actividades de aprendizaje con los criterios y niveles.

- Desarrollar un algoritmo que calcule automáticamente la calificación de cada estudiante a partir de los resultados recogidos de Moodle.

- Evaluar la coherencia entre las actividades de aprendizaje y las competencias.

- Evaluar la utilidad de las rúbricas como herramienta formativa en el contexto de la asignatura analizada. 


\section{Desarrollo de la innovación}

\subsection{Contexto}

Este trabajo se enmarca en la asignatura de Termodinámica Técnica y Fundamentos de Transmisión de Calor que se imparte en el tercer semestre ( $2^{\circ}$ curso) del Grado en Ingeniería de Tecnologías Industriales en la Universidad de Zaragoza. Cuenta con 6 ECTS, equivalentes a 150 horas de trabajo, repartidas en 45 horas de clases presenciales de teoría y resolución de problemas, 15 horas de prácticas de laboratorio y 90 horas de trabajo personal del alumno. Durante el curso 2018-2019, la asignatura cuenta con 62 alumnos matriculados.

Durante los últimos años, los recursos, metodologías y actividades de aprendizaje se han ido modificando y ampliando para conseguir un mejor aprovechamiento de las clases presenciales, tanto magistrales como de laboratorio.

En la actualidad, se dispone de:

- 3 Cuestionarios tras la visualización de Videos: 13 preguntas

- 8 Cuestionarios de autoevaluación: 102 preguntas

- 6 Formularios de respuesta de trabajos tutorizados: 85 preguntas

- 5 Cuestionarios de evaluación del prácticas: 30 preguntas

Todas estas actividades están enmarcadas en una experiencia de aula inversa (Peña, 2018), aplicada tanto en prácticas como en clases magistrales, y asociadas a un estudio de analíticas de aprendizaje basado en la información registrada en Moodle (Peña, 2019).

\subsection{Planificación del diseño de rúbricas}

Los estudios sobre el carácter formativo de las rúbricas no son concluyentes con respecto a si las rúbricas realmente mejoran el proceso de aprendizaje, ya que se pueden encontrar resultados contradictorios en la bibliografía (Jonsson, 2007; McCormick 2007; Reitmeier, 2009). De esto, se puede deducir que la correcta definición de las rúbricas y la adecuada planificación de su aplicación son complicadas, pero resultan esenciales para tener éxito.

Panadero y Jonsson analizaron 21 estudios sobre las formas en que se deben usar las rúbricas para mejorar el rendimiento de los alumnos y los factores que influyen positiva o negativamente en el proceso de aprendizaje (Panadero, 2013). Llegaron a la conclusión de que hay varias formas de mejorar el rendimiento de los estudiantes mediante el uso formativo de rúbricas: aumentar la transparencia de la evaluación para que el alumno tenga claro lo que se espera de él, reducir la ansiedad con respecto a las tareas encomendadas, facilitar la realimentación, mejorar la autoeficacia y apoyar el aprendizaje autorregulado por parte del estudiante.

Dornish y McLoughlin también habían identificado algunos factores que dificultan el uso de rúbricas con fines formativos, que en general coinciden con las observaciones anteriores: criterios demasiado generales, demasiado numerosos o poco relevantes para el peso asignado, diferencias poco claras entre los niveles de aprendizaje y descriptores demasiado generales o demasiado específicos (Dornish, 2006). 
En base a estos estudios, el procedimiento seguido para el diseño de rúbricas formativas ha sido el siguiente:

1. Seleccionar las competencias que debe desarrollar el alumno en la asignatura según el plan de estudios.

2. Definir los criterios asociados a las competencias: uno a uno, desarrollando varios criterios para cada competencia o viceversa.

3. Establecer cuatro niveles de desempeño: en proceso, básico, intermedio y avanzado.

4. Relacionar un descriptor específico por cada nivel y criterio que explique en detalle qué debe saber el alumno en cada caso. Las rúbricas se han definido de manera que el conocimiento es acumulativo, por lo que el descriptor para un cierto nivel incluye las características de los niveles previos.

Con el fin de sistematizar el proceso de evaluación por rúbricas, se ha establecido el siguiente procedimiento de calificación:

1. Seleccionar las actividades de aprendizaje que se incluirán en el proceso de evaluación por rúbricas.

2. Relacionar cada pregunta particular con uno o varios criterios y niveles de desempeño

3. Asignar un peso a cada pregunta dentro del criterio.

4. Desarrollar un algoritmo de cálculo para calcular la calificación de cada estudiante con respecto a cada criterio a partir de las puntuaciones en las preguntas relacionadas.

5. Descargar desde el VLE las calificaciones de cada actividad en hojas de cálculo y aplicar el algoritmo de rúbricas.

6. Actualizar las rúbricas en momentos clave del cuatrimestre (por ejemplo, antes de las pruebas de evaluación) e informar a los estudiantes.

7. Implementar acciones correctivas si se obtiene una calificación promedio demasiado baja en una criterio en particular.

\subsection{Ejemplo de rúbrica}

La competencia específica a desarrollar en la asignatura, tal y como figura en el plan de estudios de GITI, es (C18): "Conocimientos de termodinámica aplicada y transmisión de calor. Principios básicos y su aplicación a la resolución de problemas de Ingeniería". Los resultados de aprendizaje son un poco más concretos:

- Conoce las propiedades termofísicas de interés industrial y tiene capacidad para utilizar y seleccionar procedimientos y herramientas adecuadas para su cálculo.

- Conoce y aplica las leyes de la termodinámica al análisis energético de equipos y procesos básicos en ingeniería.

- Conoce los criterios básicos para el análisis de ciclos termodinámicos. 
- Conoce y aplica los mecanismos básicos de transferencia de calor al análisis de equipos térmicos.

- Resuelve razonadamente problemas básicos de termodinámica técnica y transferencia de calor aplicados a la ingeniería.

En base a estos resultados de aprendizaje se han definido 10 criterios, con 3 niveles de aprendizaje (más el de punto de partida). Para alcanzar cada nivel se ha establecido un umbral, que deberá ajustarse en función de los resultados para que las rúbricas resulten motivadoras. Puesto que el conocimiento es acumulativo, para alcanzar un nivel se debe dominar también lo exigido en niveles inferiores.

A modo de ejemplo, en la Tabla 1 se muestran varios de los criterios que forman parte de la rúbrica diseñada para la asignatura de Termodinámica Técnica y Fundamentos de Transmisión de Calor.

\begin{tabular}{|c|c|c|c|c|}
\hline Criterio & Aprendiz & Nivel básico (a) & Nivel intermedio (b) & Nivel avanzado (c) \\
\hline Umbral por nivel & $0 / 10$ & $6 / 10$ & $8 / 10+6 / 10$ & $8 / 19+8 / 10+6 / 10$ \\
\hline $\begin{array}{l}\text { Comprensión de } \\
\text { propiedades y procesos }\end{array}$ & Por defecto & $\begin{array}{l}\text { Conoce los tipos de } \\
\text { propiedades y procesos }\end{array}$ & $\begin{array}{l}\text { + Identifica los tipos de proceso } \\
\text { correctamente }\end{array}$ & $\begin{array}{l}\text { + Aplica las ecuaciones } \\
\text { adecuadas y los representa en } \\
\text { diagramas }\end{array}$ \\
\hline $\begin{array}{l}\text { Uso correcto del modelo de } \\
\text { Gas Ideal }\end{array}$ & Por defecto & $\begin{array}{l}\text { Utiliza correctamente la } \\
\text { ecuación térmica de estado: } \\
\text { unidades }\end{array}$ & $\begin{array}{l}\text { + Sabe calcular las variaciones de } \\
\text { energía interna, entalpía y } \\
\text { entropía }\end{array}$ & $\begin{array}{l}\text { + Aplica el modelo de Gas Ideal } \\
\text { en su contexto }\end{array}$ \\
\hline $\begin{array}{l}\text { Aplicación de los balances } \\
\text { de energía y entropía en } \\
\text { régimen estacionario }\end{array}$ & Por defecto & $\begin{array}{l}\text { Sabe aplicar los balances de } \\
\text { masa y energía }\end{array}$ & $\begin{array}{l}\text { + Sabe aplicar el balance de } \\
\text { entropía }\end{array}$ & $\begin{array}{l}\text { + Aplica los balances adecuados } \\
\text { en su contexto }\end{array}$ \\
\hline $\begin{array}{l}\text { Conocimiento de ciclos } \\
\text { termodinámicos }\end{array}$ & Por defecto & $\begin{array}{l}\text { Sabe aplicar el análisis } \\
\text { termodinámico a los ciclos } \gamma \\
\text { calcular sus prestaciones }\end{array}$ & $\begin{array}{l}\text { + Reconoce los equipos que } \\
\text { forman cada ciclo y conoce su } \\
\text { función }\end{array}$ & $\begin{array}{l}\text { + Sabe identificarlos y } \\
\text { representarlos en diagramas }\end{array}$ \\
\hline
\end{tabular}

Tabla 1. Ejemplos de algunos criterios con sus descriptores y niveles.

\section{Resultados}

Los resultados presentados en este trabajo son preliminares, pero se han podido extraer algunas conclusiones interesantes. Hasta la fecha, se han definido 10 criterios con sus niveles y descriptores y se ha desarrollado el algoritmo que calcula la calificación obtenida en cada nivel para cada criterio. Por tanto, se han completado las etapas 1-4 del procedimiento de calificación descrito en la sección anterior.

Durante el curso actual, las rúbricas han estado a disposición de los alumnos, aunque no se ha podido informar al estudiante de su progreso real, debido al tiempo requerido para el desarrollo del algoritmo. Sin embargo, se ha obtenido un mejor aprovechamiento de las clases de prácticas y un mayor porcentaje de éxito en las pruebas de evaluación (alrededor de 15 puntos porcentuales con respecto al curso 2017-2018). Dado que han sido varias las modificaciones implementadas (Peña., 2019), no se puede saber en qué medida han contribuido las rúbricas a este resultado. Con el fin de conocer la opinión de los estudiantes a este respecto, para el curso próximo se elaborará una encuesta a tal efecto. 


\subsection{Desarrollo del algoritmo de calificación automática}

Si bien inicialmente se planteó la posibilidad de desarrollar una plantilla en una hoja de cálculo, finalmente se optó por desarrollar un programa en Matlab, para que su aplicación posterior requiera menos esfuerzo. Además, es un formato más flexible con el cual implementar modificaciones es más rápido.

El algoritmo sigue el diagrama de flujo de la Figura 2. De Moodle se extraen las calificaciones de cada formulario o cuestionario en hojas de cálculo. Estos datos se leen desde un programa de Matlab, así como el listado de alumnos. Una vez establecido el alcance del cálculo en base al momento del cuatrimestre en que se aplica, se calcula la calificación numérica mediante scripts independientes para cada criterio y nivel. A partir de los umbrales que el profesor ha establecido, se asigna un nivel de progreso a cada alumno en cada criterio.

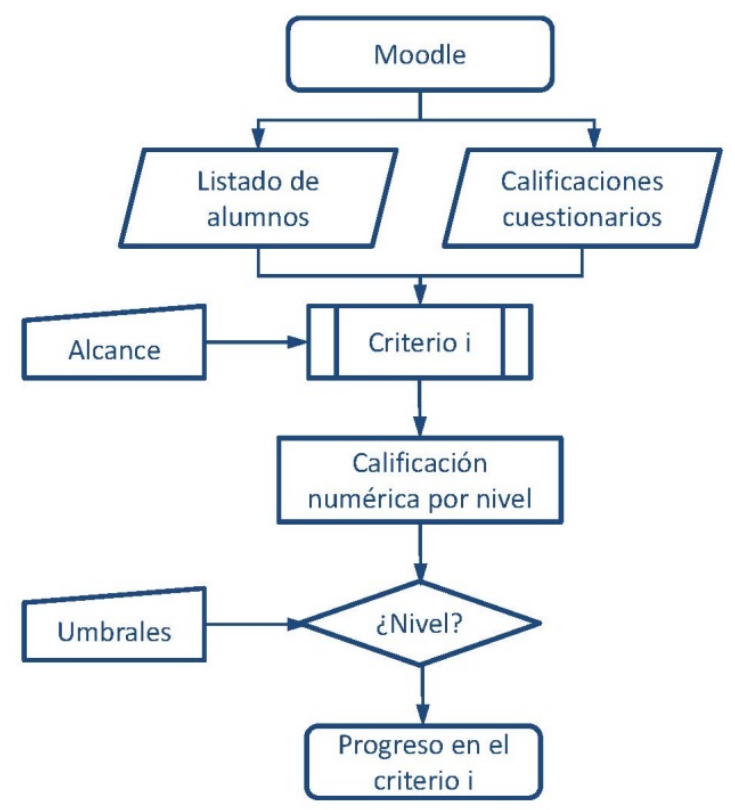

Fig. 2 Diagrama de flujo para obtener el nivel alcanzado en el criterio i de una rúbrica.

Como ya se ha comentado, para el curso actual no se ha podido utilizar este algoritmo para informar al estudiante de sus progresos a lo largo del cuatrimestre. El análisis se realizará off-line con el fin de evaluar estas rúbricas como herramienta para predecir el éxito en la evaluación final. Con este objetivo, se realizará un estudio para correlacionar la evaluación por rúbricas con las calificaciones obtenidas en las pruebas de la evaluación continua y en el examen final. 
Existe incertidumbre al respecto, ya que los hábitos de estudio son muy variables. A partir de una revisión cualitativa de los cuestionarios, se ha observado que algunos alumnos, por ejemplo algunos de los que repiten en la asignatura, apenas participan en este tipo de actividades, pero después superan con éxito las pruebas objetivas. Sin embargo, estudiantes con un número de interacciones varias veces superior a la media fallan en dichas pruebas (Peña, 2019). Si éstos últimos realizan intentos hasta obtener la máxima calificación (son ilimitados), sin haber revisado la materia para comprender por qué una respuesta es correcta y otra no lo es, esa calificación es ficticia y no será representativa del progreso real del estudiante. En este sentido convendrá informar de las limitaciones del sistema de rúbricas para que no lleve a auto-engaño.

\subsection{Evaluación de las actividades de aprendizaje}

Aunque no era el objetivo inicial del desarrollo de las rúbricas formativas, durante la definición de las mismas se tuvo conciencia de ciertas carencias en las actividades de aprendizaje en relación a las competencias que el estudiante debe adquirir y también en cuanto a la distribución de las preguntas en los cuestionarios, de cara a simplificar la evaluación mediante rúbricas automatizadas. .

El número de preguntas inicialmente asociado a cada criterio y nivel se recoge en la siguiente tabla. Algunas de las preguntas están asociadas a varios criterios a la vez, ya que son problemas cortos que conllevan varios cálculos. El peso asignado a todas las preguntas es el mismo por lo que, una vez cargados los datos correspondientes, la calificación se obtiene del promedio de las calificaciones de las preguntas individuales.

\begin{tabular}{|c|c|c|c|}
\hline Criterio & Básico & Intermedio & Avanzado \\
\hline 1 & 35 & 32 & 56 \\
\hline 2 & 25 & 15 & 22 \\
\hline 3 & 13 & 6 & 7 \\
\hline 4 & 8 & 4 & 4 \\
\hline 5 & 27 & 11 & 18 \\
\hline 6 & 9 & 10 & 20 \\
\hline 7 & 6 & 6 & 7 \\
\hline 8 & 26 & 21 & 13 \\
\hline 9 & 4 & 14 & 3 \\
\hline 10 & 9 & 3 & 3 \\
\hline
\end{tabular}

Tabla 2. Número de preguntas asociado a cada criterio y nivel.

A partir de esta tabla se realizó un análisis crítico sobre diferentes aspectos de las actividades de evaluación y sobre la asignación de preguntas a criterios, que han servido para mejorar el proceso. Se observa que existen grandes desigualdades en cuanto al número de preguntas asignadas a cada criterio y nivel. 
En algunos casos (criterios 1, 2 y 5) es natural que el número sea elevado, ya que se trata de conceptos o procedimientos esenciales para cualquier sistema termodinámico a analizar. En otros casos (criterio 10), se manifiesta el hecho de que las preguntas agrupan un problema completo y se ha planteado desglosarlo en varias preguntas o en asignarles un peso mayor dentro del criterio/nivel.

En los criterios 4, 7 y 9, sí que se ha detectado un bajo número de preguntas asociadas a algunos niveles. Para el próximo curso se añadirán nuevas preguntas para que el estudiante trabaje esa materia con mayor profundidad.

Por el momento, se ha asignado el mismo peso a todas las competencias. Sin embargo, no tienen la misma importancia dentro de la asignatura y en la evaluación final. Para el curso próximo se discutirá sobre este tema con el resto de profesores que imparten esta misma asignatura en el grado de Ingeniería de Tecnologías Industriales, con el fin de establecer pesos diferenciados.

\section{Conclusiones}

En este trabajo se ha presentado la metodología seguida para definir rúbricas cuantitativas que se pueden obtener automáticamente mediante un algoritmo basado en la huella digital que los estudiantes dejan en la plataforma Moodle. Concretamente se ha aplicado a la asignatura de "Ingeniería de Termodinámica y Fundamentos de la Transmisión de Calor", del Grado en Ingeniería de Tecnologías Industriales.

Los resultados y conclusiones se resumen a continuación:

- Aunque el objetivo inicial era utilizar las rúbricas para informar al estudiante de sus logros, también han servido para analizar y mejorar la coherencia entre las actividades de aprendizaje y las competencias a adquirir en la asignatura.

- Durante el presente curso, las rúbricas se han puesto a disposición de los estudiantes, aunque no se ha podido informar sobre su progreso a lo largo del cuatrimestre, debido al tiempo necesario para desarrollar el algoritmo de cálculo de calificaciones.

- Si bien se ha obtenido un aumento de 15 puntos porcentuales en el número de aprobados en la primera convocatoria del curso 2018-2019, no puede afirmarse que se deba enteramente al conocimiento de las rúbricas, ya que se han implementado otras innovaciones de forma simultánea (Peña, 2019).

- El análisis off-line de los resultados de este curso, permitirá analizar la capacidad de las rúbricas para predecir el éxito en la asignatura y para identificar desviaciones durante el proceso de aprendizaje, debidas tanto a un uso inapropiado de los recursos, como a ineficiencias en el planteamiento de las actividades de aprendizaje. 


\section{Agradecimientos}

La autora agradece a los profesores $\mathrm{M}^{\mathrm{a}}$ Belén Zalba Nonay, Ignacio Zabalza Bribián, Eva Llera Sastresa, Sergio Usón Gil y José María Marín Herrero su disposición para discutir y compartir experiencia en materia de innovación docente.

\section{Referencias}

DORNISH, M.M. y MCLOUGHLIN, A.S. (2006) "Limitations of web-based rubric resources. Addressing the challenges", Practical Assessment, Research \& Evaluation, vol. 11, issue 3. Available on-line: http://pareonline.net/getvn.asp?v=11\&n=3. (Constulta: 22 de marzo de 2019).

JONSSON, A. y SVINGBY, G. (2007) "The use of scoring rubrics: Reliability, validity and educational consequences”, Educational Research Review, vol. 2, pp. 130-144.

McCORMICK, M.J., DOOLEY, K.E., LINDNER, J.R. y CUMMINS, R.L. (2007) "Perceived growth versus actual growth in executive leadership competencies: An application of the stair-step behaviorally anchored evaluation approach", Journal of Agricultural Education, vol. 48, issue 2, p. $23-35$.

MOODLE (2014) "Rubrics", Moodle Documentation - Advanced grading methods. URL: https://docs.moodle.org/22/en/Rubrics. (Constulta: 22 de marzo de 2019).

PANADERO, E. y JONSSON, A. (2013) "The use of scoring rubrics for formative assessment purposes revisited: a review”, Educational Research Review, vol 9, p. 129-144.

PEÑA, B. et al. (2018) "Experiencia piloto de aula invertida para mejorar el proceso de enseñanzaaprendizaje en la asignatura de Termodinámica Técnica". En: Actas del congreso INRED 2018, Valencia, Editorial Universitat Politècnica de València. Doi: http://dx.doi.org/10.4995/INRED2018.2018.8583. Disponible en: < http://ocs.editorial.upv.es/index.php/INRED/INRED2018/paper/viewFile/8583/4153> [Consulta : 9 de febrero de 2019].

PEÑA, B. (2019) "Análisis del aprendizaje a través de la huella en Moodle: aplicación en la asignatura de Termodinámica Técnica” En: Actas del congreso INRED 2019, Valencia, Editorial Universitat Politècnica de València. (Enviado: 14 de marzo de 2019)

REDDY, Y.M. y ANDRADE, H. (2010) “A review of rubric use in higher education”, Assessment \& Evaluation in Higher Education, vol. 35, issue .4, p. 435-448. DOI: 10.1080/02602930902862859.

REITMEIER, C.A. y VRCHOTA, D.A. (2009) "Self-assessment of oral communication presentations in food science and nutrition", Journal of Food Science Education, vol. 8, issue 4, p. 88-92. 